\section{Improved Procedure for Examination of Gap Junctional Intercellular Communication by In Situ Electroporation on a Partly Conductive Slide}

\section{BioTechniques 29:222-226 (August 2000)}

Gap junctions are plasma membrane channels that serve as conduits for the passage of small molecules and ions between the interiors of cells (6). Gap junctional intercellular communication (GJIC) has long been thought to play an important role in cell growth and differentiation, as well as in the metastatic potential of human cancer cells $(6,23)$. The investigation of junctional permeability is usually conducted through the introduction of a fluorescent dye such as Lucifer yellow (LY), through microinjection, scrape-loading or preloading (13), followed by observation of its migration into neighboring cells or by measuring the recovery of fluorescence after photobleaching (22). These methods are expensive and time consuming; they may also introduce the potential complication of cellular damage.

We have developed a novel technique for GJIC measurement that can overcome these problems (17). This technique, termed in situ electroporation on a partly conductive slide, takes advantage of the ability of short electric pulses to create transient "pores" on the cell membrane through which LY can enter simultaneously into large numbers of cells with minimal disturbance to cellular physiology $(7,16-18)$.

Cells are grown on a glass slide, half of which is coated with electrically conductive, optically transparent indium-tin oxide (Applied Films, Boulder, CO, USA). The nonconductive area is created by removing the conductive coating with hydrochloric acid in the presence of metallic zinc powder, thus exposing the bare glass underneath $(9,17,19)$. An electric pulse is applied in the presence of LY, causing its penetration into the cells growing on the conductive half of the slide, and the migration of the dye through gap junctions to the non-electroporated cells growing on the nonconductive area is microscopically observed under fluorescence illumination (17) (Figure 1, A and B, and Figure 2). In this way, dye transfer through gap junctions can be precisely quantitated simultaneously and in a large number of cells, without any detectable disturbance to the cell $(2,18)$. At the same time, contrary to microinjection or scrape-loading (8), there is no mechanical manipulation of the cells that might disturb cell-to-cell contact areas, interrupt gap junctions and cause artifactual uncoupling. This approach, which offers the ability to quantitate GJIC, made possible the examination of the link between levels of oncogene expression, transformation

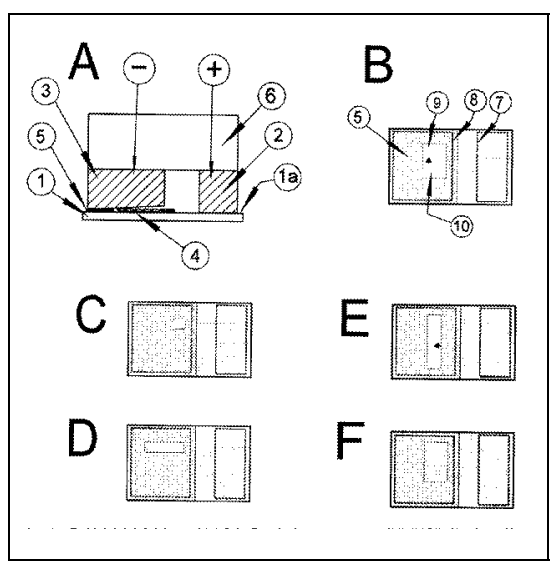

Figure 1. Electrode and slide assembly for the study of intercellular, junctional communication. (A) Side view: a coating of indium-tin oxide [1a] on the upper surface of the glass slide [1] makes a conductive path from the positive contact bar [2] through the cells [4] and the electroporation solution to the bottom surface of the negative electrode [3]. The + and - signs denote connections to the positive and negative poles of the pulse source. (B) Top view of standard slide: the outline of the positive [7] and negative [8] electrodes and their relative position on the slide in relation to the frame [5] and the window where the cells are grown on both the conductive and nonconductive areas $[9,10]$ are indicated. The lightly shaded area [10] represents the conductive coating, and the arrowhead points to the transition line between conductive and nonconductive areas. (C) Same as in B, window of $2 \times 2 \mathrm{~mm}$. (D) Same as in $\mathrm{B}$, window of $4 \times 15 \mathrm{~mm}$ with its long axis parallel to the length of the slide. (E) Top view of transverse-etched slide: a narrow window confines the cells in the region of the conductive [10] to nonconductive [9] transition line that runs laterally across the slide. (F) Top view of stripeetched slide: the conductive coating [10] has been removed in $1 \mathrm{~mm}$ wide strips [9]. This produces a longer length of conductive to nonconductive transition line along which the transfer of LY, and hence gap junctions, can be observed. and GJIC, as well as observation of gap junction closure upon adipocyte differentiation $(3-5,17)$. This technique was later adapted for GJIC examination of lines established from human lung carcinomas or cells freshly explanted from lung-tumor tissues $(20,21)$.

Senescence of human umbilical vein endothelial cells after a number of passages in culture induces a reduction in GJIC (24). During culture of human lung carcinoma cells $(20,21)$, it became evident that, contrary to the fibroblasts present in the specimen, the tumor cells senesced and died within 10-15 days. Therefore, since senescence may downregulate GJIC, to obtain a true measurement of their junctional communication in vivo, GJIC must be examined within a few days after surgery, as soon as cells have attached and before they approach their limits of life in culture. This is especially important because the (normal) fibroblasts that invariably predominate after the tumor cells have died display extensive GJIC, which makes GJIC assessment of the tumor cells problematic (21).

The slide configuration previously employed for GJIC examination consists of a window of $7 \times 4 \mathrm{~mm}$ with a conductive area of $4 \times 4 \mathrm{~mm}$ where the cells are grown, so that the transition line between conductive and nonconductive sides runs through the middle of the slide and is $4 \mathrm{~mm}$ long (17) (Figure $1 \mathrm{~B})$. This configuration is satisfactory for GJIC examination in most established cell lines. However, many cells are required because the cells need to be situated precisely at the transition line, which is a relatively small proportion of the total area, for examination of their communication to be possible. Therefore, for GJIC assessment when small amounts of tissue are available, the chances of the cells attaching at this line must be increased so that GJIC can be examined before they have divided in culture and senesced. This paper describes electrode and slide configurations that can be effectively employed for this purpose.

The use of a very small window $(2 \times$ $2 \mathrm{~mm}$, with a transition line of $2 \mathrm{~mm}$ in length and conductive area of $1 \times 2$ $\mathrm{mm}$ ) (Figure $1 \mathrm{C}$ ), requires very low voltage and capacitance settings that cannot be provided by the commercial- 
ly available pulse generators. In addition, because the voltage required for efficient permeation is proportional to the area being electroporated, a very small conductive area results in a system that is overly sensitive to small variations in conductive area size, resulting from variations in the position of the frame relative to the transition line. The use of a larger conductive area in an elongated window, with the transition line along its length, would help mitigate the problem of the small size of the conductive area being sensitive to voltage settings. However, in this case the surface resistivity of the thin conductive coating can make it difficult to obtain a uniform electric field intensity over the entire cell growth area (18). The current in situ electroporation system compensates for the conductive coating surface resistivity by placing the electrode at a shallow angle relative to the coating (3) (Figure 1A). This results in a fluid-filled gap between the conductive coating and the negative electrode that begins relatively wide at the point near the positive electrode and tapers off to a smaller gap along the length of the slide away from the positive electrode. The net effect is that the combined resistance of the fluid-filled gap and the coating along the surface produces an electric field sufficiently uniform to electroporate the entire area of exposed cells when subject to a pulse. With a slide configured as shown in Figure 1D, we found that slight variations in the conductivity of the coated glass and the parallel alignment of the frame to the transition line could result in inconsistent electroporation along the length of the transition line.

Two slide configurations that successfully address the problems described above are shown in Figure 1, E and $\mathrm{F}$. In Figure 1E, the conductive coating was removed from the distal part of the slide, and the frame was placed as shown. In this setup, because the distance difference between the proximal and distal ends of the conductive surface relative to the positive electrode is only about $2 \mathrm{~mm}$, it is very easy to achieve a uniform electrical field over the entire area and along the transition line. However, when it is made narrow enough to force the cells to grow along the transition line, the long, thin lateral window format still demands a precise placement of the frame (parallel to the transition line, within a tenth of a millimeter over the width of the window).

An alternative setup is described in Figure $1 \mathrm{~F}$, in which the conductive coating was removed in a pattern of $1 \mathrm{~mm}$ wide strips. In this configuration, although the etching process is somewhat more complicated, the precise positioning of the frame is not essential because the size of the conductive growth area is determined by the thickness of the strips and the distance between them. For either window type (Figure 1, E or F), the width of the conductive strip should be at least equal to the length of two cells, while the width of the nonconductive part must be greater than the greatest possible distance traveled by the dye. For rat F111 cells that have extensive GJIC (17), a width of $1 \mathrm{~mm}$ is sufficient in both respects.

Figure 3 shows the relationship between pulse conditions and the amount of dye loaded using the assemblies described in Figure 1, B, C and E. Human lung carcinoma QUDB cells (21) were electroporated in the presence of 2 $\mathrm{mg} / \mathrm{mL} \mathrm{LY}$, prepared in PBS at $0.1 \mu \mathrm{F}$ and different voltage settings. After washing the unincorporated LY with serum-containing DMEM, the cells were lysed and the relative amount of LY introduced quantitated by fluorescence measurements using a model 204A fluorescence spectrophotometer (excitation $428 \mathrm{~nm}$, emission $540 \mathrm{~nm}$ ) (19) (PE Biosystems, Foster City, CA, USA). As shown in Figure 3, the voltage and capacitance settings required for efficient penetration depended mainly on the area electroporated, irrespective of the etching pattern of the cell growth surface. In addition, for this capacitance setting, the margins of voltage tolerance, which are invariably wider for the introduction of LY compared to larger molecules (16), are greater for the larger areas electroporated $(15 \times 2 \mathrm{~mm})$.

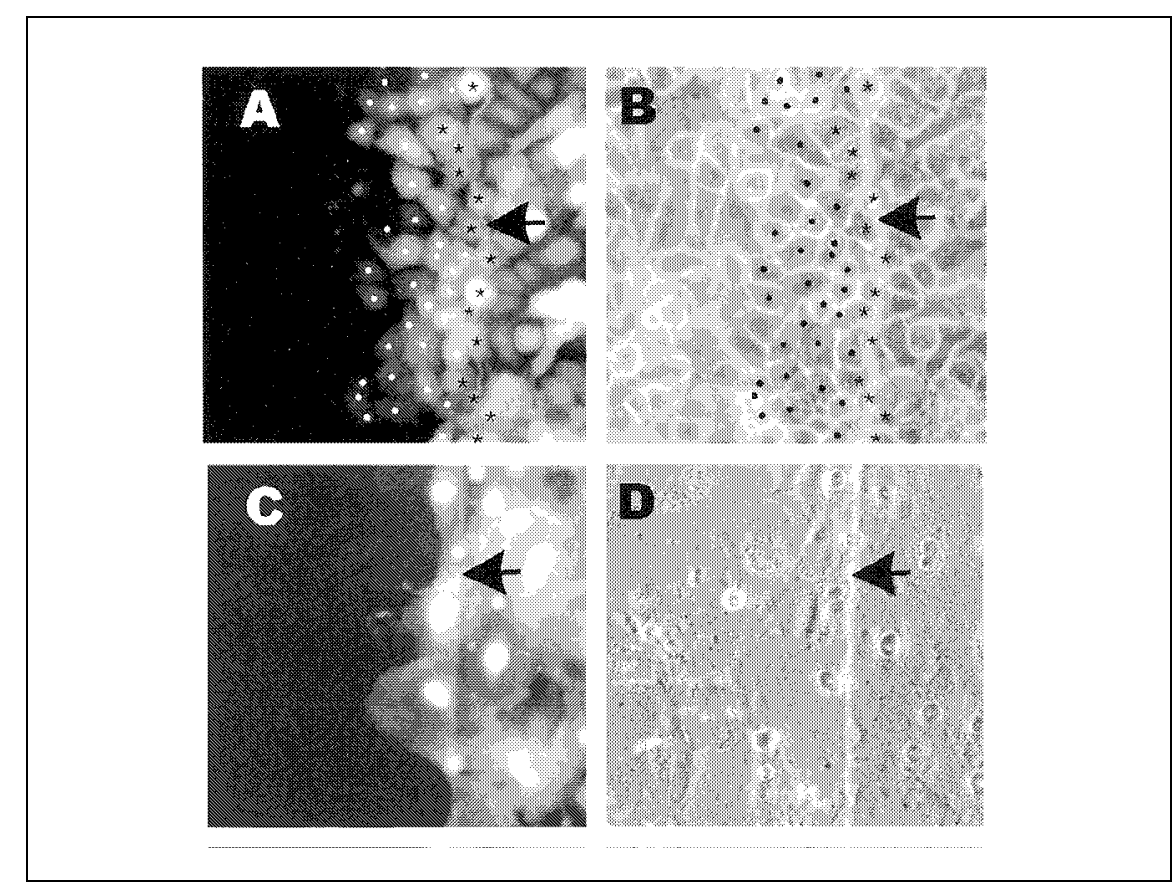

Figure 2. Senescence interrupts junctional communication. Cells from tumors explanted from the lungs of $\mathrm{A} / \mathrm{J}$ mice 18 weeks after injection of urethane were plated on slides such as those described in Figure 1E. Three days later, cells were electroporated in the presence of $2 \mathrm{mg} / \mathrm{mL}$ LY. Five minutes after the pulse, unincorporated dye was washed, and cells from the same field were photographed under fluorescence (A) or phase contrast (B) illumination. Parallel cultures were grown for 40 days after surgery, then plated on the same type of slide and electroporated (C and D). To quantitate intercellular communication, the number of cells into which the dye has transferred through gap junctions (circles) can be divided by the number of electroporated border cells (stars). Arrows on the conductive side point to the transition line between conductive and nonconductive areas. This line is visible under fluorescence because of dye accumulation. Note the extensive communication present in cells immediately after surgery (A) compared to cells tested 40 days later (C). These cells display a very extended (flat) morphology that is typical of cells approaching senescence. 
The results of GJIC examination using the assembly described in Figure 1E are shown in Figure 2. In this experiment, GJIC was measured in urethaneinduced lung carcinomas in $\mathrm{A} / \mathrm{J}$ mice. In this animal model, 1-2-mm tumors appear in the lungs approximately 18 weeks after injection of urethane to one-month-old mice ( $1 \mathrm{mg} / \mathrm{kg}$, i.p.) (10, 11). These tumors were dissected, and cells were plated directly on the slides (Figure 1E). Cells rapidly attached, and GJIC could be measured three days later. As shown in Figure 2A, these cells have extensive GJIC, indicating that junctional permeability is still high at this stage in tumor development (manuscript in preparation). However, after 40 days in culture, these cells were found to have lost their junctional communication, probably because of the senescence process (Figure 2C).

In situ electroporation on a partly conductive slide is a powerful technique for GJIC examination. The refinements described should enable rapid GJIC examination using small amounts of tissue such as biopsies for use in many applications including cancer diagnosis or prognosis. The same setup can be used for the introduction of other nonpermeant molecules, such as prodrugs and peptides, into such cells for a variety of biochemical applications $(1,2,12,14,15)$.

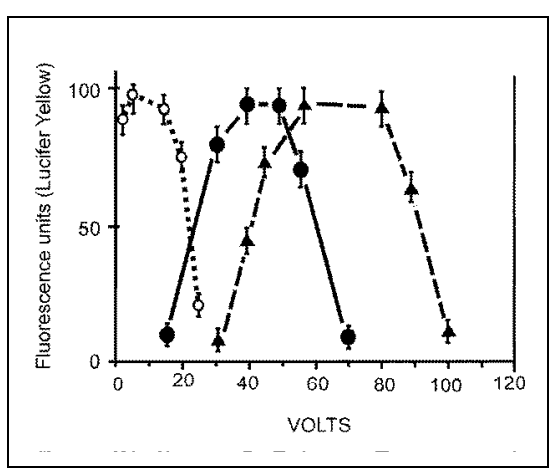

Figure 3. Effect of field strength upon the introduction of LY into cells grown on ITO-coated slides with the coating removed in different patterns. Human lung carcinoma QUDB cells were electroporated in the presence of $2 \mathrm{mg} / \mathrm{mL}$ $\mathrm{LY}$ at a capacitance of $0.1 \mu \mathrm{F}$ and different voltage settings. After washing of the unincorporated LY, cells were lysed and the relative amount of LY introduced quantitated by fluorescence measurement. (o): conductive area, $2 \times 1 \mathrm{~mm}$ (Figure 1C). (•); conductive area, $4 \times 4 \mathrm{~mm}$ (Figure 1B). (४): conductive area, $15 \times 2 \mathrm{~mm}$ (Figure 1E).

\section{REFERENCES}

1.Boccaccio, C., M. Ando, L. Tamagnone, A. Bardelli, P. Michielli, C. Battistini and P.M. Comoglio. 1998. Induction of epithelial tubules by growth factor HGF depends on the STAT pathway. Nature 391:285-288.

2.Brownell, H.L., N. Lydon, E. Schaefer, T.M. Roberts and L. Raptis. 1998. Inhibition of epidermal growth factor-mediated ERK1/2 activation by in situ electroporation of nonpermeant [(alkylamino)methyl]acrylophenone derivatives. DNA Cell Biol. 17:265-274.

3.Brownell, H.L., R. Narsimhan, M.J. Corbley, V.M. Mann, J.F. Whitfield and L. Raptis. 1996. Ras is involved in gap junction closure in mouse fibroblasts or preadipocytes but not in differentiated adipocytes. DNA Cell Biol. 15:443-451.

4.Brownell, H.L., J.F. Whitfield and L. Raptis. 1996. Cellular Ras partly mediates gap junction closure by the polyoma virus middle Tumor antigen. Cancer Lett. 103:99-106.

5.Brownell, H.L., J.F. Whitfield and L. Raptis. 1997. Elimination of intercellular junctional communication requires lower Ras ${ }^{\text {leu61 }}$ levels than stimulation of anchorage-independent proliferation. Cancer Detect. Prev. 21:289-294.

6.Bruzzone, R., T.W. White and D.L. Paul. 1996. Connections with connexins: the molecular basis of direct intercellular signalling. Eur. J. Biochem. 238:1-27.

7.Chang, D.C., B.M. Chassy, J.A. Saunders and A.E. Sowers. 1992. Guide to Electroporation and Electrofusion. Academic Press, San Diego.

8.el-Fouly, M.H., J.E. Trosko and C.C. Chang. 1987. Scrape-loading and dye transfer: a rapid and simple technique to study gap junctional intercellular communication. Exp. Cell Res. 168:422-430.

9.Firth, K.L., H.L. Brownell and L. Raptis. 1997. Improved procedure for electroporation of peptides into adherent cells in situ. BioTechniques 23:644-645

10.Forkert, P.G., J.A. Lord and A. Parkinson. 1996. Alterations in expression of CYP1A1 and NADPH-cytochrome P450 reductase during lung tumor development in SWR/J mice. Carcinogenesis 17:127-132.

11.Forkert, P.G., A. Parkinson, L.G. Thaete and A.M. Malkinson. 1992. Resistance of murine lung tumors to xenobiotic-induced cytotoxicity. Cancer Res. 52:6797-6803.

12.Giorgetti-Peraldi, S., E. Ottinger, G. Wolf, B. Ye, T.R. Burke and S.E. Shoelson. 1997. Cellular effects of phosphotyrosine-binding domain inhibitors on insulin receptor signalling and trafficking. Mol. Cell. Biol. 17:1180-1188.

13.Goldberg, G.S., J.F. Bechberger and C.C. Naus. 1995. A pre-loading method of evaluating gap junctional communication by fluorescent dye transfer. BioTechniques 18:490-497.

14.Haruta, T., A.J. Morris, P. Vollenweider, J. Nelson, D.W. Rose, M. Mueckler and J.M. Olefsky. 1998. Ligand-independent Glut4 translocation induced by guanosine 5'-O-(3-thiophosphate) involves tyrosine phosphorylation. Endocrinology 139:358-364.

15.Marais, R., R.A. Spooner, S.M. Stribbling, Y. Light, J. Martin and C.J. Springer. 1997. A cell surface tethered enzyme improves efficiency in gene-directed enzyme prodrug therapy.
Nat. Biotechnol. 15:1373-1377.

16.Raptis, L., H.L. Brownell, K.L. Firth and S. Giorgetti-Peraldi. 1998. In situ electroporation for the study of signal transduction, p. 7587. In J.C. Celis (Ed.), Cell Biology: A Laboratory Handbook. Academic Press, San Diego.

17.Raptis, L., H.L. Brownell, K.L. Firth and L.W. MacKenzie. 1994. A novel technique for the study of intercellular, junctional communication; electroporation of adherent cells on a partly conductive slide. DNA Cell Biol. 13: 963-975.

18.Raptis, L. and K.L. Firth. 1990. Electroporation of adherent cells in situ. DNA Cell Biol. 9:615-621.

19.Raptis, L.H., S.K.W. Liu, K.L. Firth, C.D. Stiles and J.A. Alberta. 1995. Electroporation of peptides into adherent cells in situ. BioTechniques 18:104-114.

20.Tomai, E., H.L. Brownell, T. Tufescu, K. Reid and L. Raptis. 1999. Gap junctional communication in lung carcinoma cells. Lung Cancer 23:223-231.

21.Tomai, E., H.L. Brownell, T. Tufescu, K. Reid, S. Raptis, B.G. Campling and L. Raptis. 1998. A functional assay for intercellular, junctional communication in cultured human lung carcinoma cells. Lab. Invest. 78:639-640.

22.Wade, M.H., J.E. Trosko and M. Schindler. 1986. A fluorescence photobleaching assay of gap junction-mediated communication between human cells. Science 232:525-528.

23.Weinstein, R.S., F.B. Merk and J. Alroy. 1976. The structure and function of intercellular junctions in cancer. Adv. Cancer Res. 23:23-89.

24.Xie, H.Q., R. Huang and V.W. Hu. 1992. Intercellular communication through gap junctions is reduced in senescent cells. Biophys. J. 62:45-47.

This work was supported by grants from the Natural Sciences and Engineering Research Council of Canada, from the Cancer Research Society Inc. and the Advisory Research Committee of Queen's University to L.R., and a grant from the Medical Research Council of Canada to P.G.F. E.T. is the recipient of a Queen's University Graduate Award, an Ontario Graduate Studentship in Science and Technology, an award from the Ontario Thoracic Society and a scholarship from the M.P. Lemos foundation. Addresscorrespondence to Dr. Leda Raptis, Dept. of Microbiology and Immunology and Dept. of Pathology, Queen's University, Kingston, ON, Canada, K7L 3N6. e-mail: raptisl@ post.queensu.ca

Received 8 November 1999; accepted 14 April 2000.

Leda Raptis, Kevin L. Firth', Evangelia Tomai and Poh-Gek Forkert

Queen's University and

${ }^{1}$ ASK Science Products

Kingston, ON, Canada 